\title{
Control of Flexible Systems in the Presence of Failures
}

\author{
Peiman G. Maghami * \\ NASA Goddard Space Flight Center, Greenbelt, MD 20771 \\ David E. Cox ${ }^{\dagger}$ \\ NASA Langley Research Center, Hampton, VA 23681
}

\begin{abstract}
Control of flexible systems under degradation or failure of sensors/actuators is considered. A Linear Matrix Inequality framework is used to synthesize $H_{\infty}$-based controllers, which provide good disturbance rejection while capable of tolerating real parameter uncertainties in the system model, as well as potential degradation or failure of the control certainties in the system model, as well as potential degre scenario is considered, wherein no more than one sensor or actuator is allowed to fail at any given time. A numerical example involving control synthesis for a two-dimensional flexible system is presented to demonstrate the feasibility of the proposed approach.
\end{abstract}

\section{Introduction}

Control design for flexible systems is especially challenging because these systems have a large number of modes with inherently small damping, some of which may reside within the desired control bandwidth. To exacerbate the problem further, the parameters associated with flexible systems, which are typically in modal form, are subject to varying degree of uncertainty. These include the modal frequencies, damping ratios, and shapes. A robust control design must take into account these uncertainties, which are generally modeled as parametric and/or non-parametric model uncertainties. Parametric uncertainties include the model uncertainties for those modes that are included in the model, whereas non-parametric uncertainties cover model uncertainties associated with the truncated modes. Flexible system control must provide at the least robust stability, and preferably, robust performance. To date, numerous works have been published in the literature dealing with various aspects of robust control of flexible systems. ${ }^{13}$ Control design methods, such as $H_{\infty}$, Structured Singular Value Approach $(\mu)$, and dissipative and passive techniques have provided a systematic framework for design of robust controllers for flexible systems.

The problem considered here goes beyond robust control of flexible systems in that a control design methodology is sought to not only provide robustness against model uncertainties, but also accommodate control system hardware failures, such as sensor or actuator failures. Generally, sensor/actuator failure

\footnotetext{
-Senior Engineer, Flight Dynamics and Control Branch, Senior Member AIAA.

${ }^{\dagger}$ Research Engineer, Guidance and Control Branch.

Copyright (c) 2001 by the American Institute of Aeronautics nnd Astro

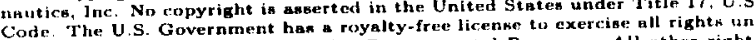
der the copyright claimed herein for Governmental Purposes. All other right are remerved by the copyright owner.
}

can be divided into soft failures or hard failures. Soft failure includes a partial loss of the instrument, resulting in an input-output degradation, i.e., a gain and phase change in the dynamics of the instrument. Hard failure refers to the total loss of the instrument. Since there is lack of methodology to deal with both soft and hard failures in a unified setting, control design and accommodation for each have followed different paths. Soft failures have traditionally been accommodated through control design with sufficient stability guarantees. The stability guarantees for single-input/single-output systems are typically handled through appropriate phase and gain margins. For multi-input/multi-output systems, the stability margins required to deal with soft sensor/actuator failures are attained through robust control design. ${ }^{1,4}$ As for hard failures, they have been typically handled through introducing hardware redundancies in the design. As one instrument fails, the fault detection and isolation system identifies the failing instrument, and the control logic replaces the instrument with a fresh redundant one. Of course, the fault detection and isolation is not an easy task. Moreover, guaranteeing the system's safe behavior during the detection, isolation, and accommodation phases is even harder.

This paper presents a methodology for the unified treatment of soft and/or hard failure of sensors/actuators in presence of model uncertainties. A Linear Matrix Inequality framework is used to synthesize $H_{\infty}$-based controllers, which provide good disturbance rejection while capable of tolerating real parameter uncertainties in the system model, as well as potential degradation or failure (soft and/or hard failure) of the control system hardware. Here, only actuator failure is considered, as the treatment of sensor failure would be similar. In this approach, a one-at-a-time failure scenario is considered, wherein no more than 
one sensor or actuator is allowed to fail at any given time. This is reasonable approach considering that for most aerospace systems the chance of concurrent multiple failures are very remote. A numerical example involving control synthesis for a two-dimensional flexible system is presented to demonstrate the feasibility of the proposed approach.

\section{Plant Description}

The dynamics of a typical flexible system are represented by a linear, time-invariant second-order system for the purpose of control design. This includes flexible spacecraft in the so-called science mode, wherein fine pointing is maintained to enable science measurements. The order of this system can be in thousands or tens of thousands of states. However, following model reduction procedures (e.g., modal truncation, modal cost analysis, etc.) the order of the system can be re duced to a manageable size. Finally, the dynamics of the flexible system is rewritten is a first-order statespace form, i.e,

$$
\begin{gathered}
\dot{x}=\bar{A} x+\bar{B} u+\bar{H} p \\
y=\bar{C} x \\
y_{p}=\bar{L} x+\bar{D}_{u} u+\bar{D}_{p} p
\end{gathered}
$$

where $\bar{A}$ denote the state matrix, matrices $\bar{B}, \bar{H}, \bar{C}$, and $\bar{L}$, represent the influence matrices for the control inputs, disturbances, measurement outputs, and performance outputs, respectively. The matrices $\bar{D}_{u}$, and $\bar{D}_{p}$, represent feed-through matrices associated with the performance outputs; $x$ denotes the state vector; $y$ denotes the measurement output vector; $y_{p}$ denotes the performance output vector; and $p$ represents the exogenous disturbance vector.

In most cases, the dynamics of the flexible system can be written in modal coordinates. This means that the modal frequency and damping information is isolated in the state matrix, while the modal amplitudes are isolated in the input/output influence matrices. Typically, there is uncertainty associated with all modal parameters. However, the uncertainty in the modal frequency, and to some degree the modal damping, is of a more critical importance in robust control design. Hence, in this paper, the model uncertainty is mainly characterized by real parameter uncertainties in the state matrix $\bar{A}$, i.e,

$$
\bar{A}=\bar{A}(\theta) ; \quad \theta=\left[\begin{array}{lll}
\theta_{1} & \ldots & \theta_{s}
\end{array}\right]
$$

where $\theta$ is the uncertainty parameter vector, whose elements are bounded as follows.

$$
\theta_{i} \epsilon\left[\begin{array}{ll}
\theta_{i}^{L} & \theta_{i}^{U}
\end{array}\right]
$$

As mentioned previously, the paper considers the degradation and/or failure of sensors and actuators. This degradation/failure may take the form of a gain variation, which affects the elements of the input/output influence matrices, or it may take the form of a phase variations, which would introduce additional dynamics into the system. In general, hardware failure would bring about a combination of gain and phase variations. Here, it is assumed that the variations in the hardware (due to degradation) can be modeled as a linear augmentation to the system dynamics. Furthermore, for simplicity of presentation the paper considers actuator failure case only. The sensor failure problem may be handled in a very similar fashion. Additional dynamics are augmented to the plant dynamics to account for potential uncertainties in the actuators. The actuator uncertainty dynamics is added to the nominal actuator dynamics (if any) and combined with the plant. The combined dynamics is given by

$$
\dot{z}=A z+B w+H p
$$

$$
y=C z
$$

$$
y_{p}=L x+D_{w} w+D_{p} p
$$

where $z=\left\lceil\begin{array}{c}x \\ x_{a}\end{array}\right.$ denotes the combined state. The state matrix and influence matrices are obtained from the series connection of the plant dynamics (Eq. (1$3)$ ) with the actuator uncertainty weighting dynamics given by

$$
\begin{gathered}
\dot{x}_{a}=A_{a} x_{a}+B_{a} w \\
u=C_{a} x_{a}+D_{a} w
\end{gathered}
$$

The order of the actuator uncertainty weighting dynamics would depend on the degree of gain and phase variations one desires to account for due to degradation.

\section{Control Synthesis}

Control system design for flexible systems is challenging because of the their special dynamic characteristics: a number of structural modes within the controller bandwidth; low, closely spaced modal frequencies; very small inherent damping; and insufficient knowledge of the parameters. For a control design to be considered feasible, it must (i) be of reasonably low order; (ii) satisfy the nominal performance specification; and (iii) be robust to errors in the design model. A feasible control design must be robust to parametric and non-parametric uncertainties in the system model. In this paper, the errors or failures in the control system hardware, such as actuators, as 
well as uncertainties in modal parameters are considered. Specifically, uncertainties in modal frequencies (which are typically the most critical) are considered. Although, sensor degradation/failure is not considered here, its treatment follows exactly that of actuator degradation/failure problem. It is desirable to design a controller that can accommodate, to a specified degree, the failure and/or degradation of the control system hardware, while providing robust performance against uncertainties in modal frequencies.

The basis of the control synthesis is an $H_{\infty}$ design. A block diagram of the closed-loop system, indicating the feedback structure of the plant, controller, and uncertainty are shown in Figure 1. The performance requirement is defined in terms of the weighted output sensitivity transfer function from disturbances to the performance output . The weighting function $W_{p}$ is typically chosen for each channel to provide good disturbance rejection at low to mid frequencies as well as to provide integral action to minimize steady-state errors. An additional uncertainty in the system model in the form of unstructured input multiplicative is used. The weighting function $W_{u}$, used to scale or distribute the uncertainty, is chosen to emphasize model uncertainty in the mid-high frequency range. This uncertainty block not only provides for perturbations in the model, but is also serves to pose a proper standard $H_{\infty}$ control problem. The standard $H_{\infty}$ design is to minimize the weighted output sensitivity transfer function from disturbances to the performance outputs, while providing robustness to the input multiplicative uncertainty as well. Now, if the purpose of the control design was to solve this standard $H_{\infty}$ problem, a controller could be synthesized using a number of available tools. However, here the controller has to not only satisfy the $H_{\infty}$ problem, it also has to allow for real structured uncertainties in the state matrix (to account for uncertainties in the modal frequencies and damping). Moreover, it has to be able to accornmodate degradations and/or faults in the actuators. In order to accommodate the various requirements of the proposed control design, a Linear Matrix Inequality (LMI) framework is used. The LMI framework offers many advantages 5

- LMI-formulated problems can be solved exactly via convex optimization algorithms

- LMI formulations can accommodate a variety of design specifications and constraints concurrently.

- Efficient convex algorithms are available

The $H_{\infty}$ control problem can be formulated in an LMI setting as follows ${ }^{6}$

$$
\min _{X, Y, \hat{A}, \hat{B}, \hat{C}, \hat{D}, \gamma, \beta} \gamma
$$

s.t.

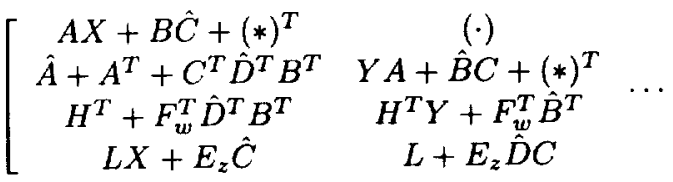

$$
\begin{aligned}
& \left.\begin{array}{cc}
(\cdot) & (\cdot) \\
(\cdot) & (\cdot) \\
-\gamma I_{w} & (\cdot) \\
D_{z w}+E_{z} \hat{D} F_{w} & -\gamma I_{z}
\end{array}\right]<0 \\
& \underset{\beta I}{\lceil} \stackrel{Y}{Y}(\cdot)\rceil<0 \\
& \beta \geq \beta_{\text {des }}
\end{aligned}
$$

where terms $(*)^{T}$ and $(\cdot)$ are implied by symmetry, matrices $X$ and $Y$ are partition matrices related to the Lyapunov matrix; $\hat{A}, \hat{B}, \hat{C}$, and $\hat{D}$, are the controller matrices in a nonlinearly transformed coordinates; ${ }^{6} \gamma$ is a real scalar whose minimum value denotes the $H_{\infty}$ norm; and $\beta$ is a scaling parameter used to avoid potential conditioning problems for the matrix $I-X Y$, whose inversion is required in realizing the controller in the physical space. $\beta_{\text {des }}$ is a threshold value larger than 1 . The $H_{\infty}$ LMI problem may also be recast from a convex minimization problem to a convex feasible solution problem, as follows

$$
\text { find } X, Y, \hat{A}, \hat{B}, \hat{C}, \hat{D}, \gamma, \beta
$$

s.t.

$$
\left.\begin{array}{ccc}
A X++B \hat{C}+(*)^{T} & (\cdot) \\
\hat{A}+A^{T}+C^{T} \hat{D}^{T} B^{T} & Y A+\hat{B} C+(*)^{T} \\
H^{T}+F_{w}^{T} \hat{D}^{T} B^{T} & H^{T} Y+F_{w}^{T} \hat{B}^{T} & \\
L X+E_{z} \hat{C} & L+E_{z} \hat{D} C \\
& (\cdot) & (\cdot) \\
& (\cdot) & (\cdot) \\
& -\gamma I_{w} & (\cdot) \\
D_{z w}+E_{z} \hat{D} F_{w} & -\gamma I_{z}
\end{array}\right]<0
$$

Here $\gamma_{\text {des }}$ represents the desired $H_{\infty}$ norm of the closed-loop system. 


\section{Model Uncertainty}

As mentioned earlier, there are real parametric uncertainties in the plant state matrix which represents uncertainties in the modal frequencies and damping. An LMI framework is an ideal setting to deal with such a problem. The problem is to find a controller $(\hat{A}$, $\hat{B}, \hat{C}, \hat{D}$ ) such that the LMI problems in Eqs. (11)(19) are satisfied with the uncertain plant matrix $A(\theta)$. Since, matrix $A(\theta)$ appears in an affine form in these LMIs, then the LMIs would be identically satisfied for all values of the uncertain parameters $\theta$ if and only if they are satisfied on the vertices of the hypercube formed by the lower bound and upper bound values of $\theta_{i}, i=1, \ldots, s$. Therefore, the LMI formulation to provide robustness against the parametric uncertainties may be written as

$$
\text { find } X, Y, \hat{A}, \hat{B}, \hat{C}, \hat{D}, \gamma, \beta
$$

s.t.

$$
\left[\begin{array}{ccc}
A\left(\theta_{\ell}\right) X+B \hat{C}+(*)^{T} & (\cdot) & \\
\hat{A}+A^{T}\left(\theta_{\ell}\right)+C^{T} \hat{D}^{T} B^{T} & Y A\left(\theta_{\ell}\right)+\hat{B} C+(*)^{T} & \\
H^{T}+F_{w}^{T} \hat{D}^{T} B^{T} & H^{T} Y+F_{w}^{T} \hat{B}^{T} & \\
L X+E_{z} \hat{C} & L+E_{z} \hat{D} C & \\
& (\cdot) & (\cdot) \\
& (\cdot) & (\cdot) \\
& -\gamma I_{w} & (\cdot) \\
& D_{z w}+E_{z} \hat{D} F_{w} & -\gamma I_{z}
\end{array}\right]<0
$$

$$
\begin{gathered}
\underset{\beta I}{X} \quad{ }^{X}(\cdot)<0 \\
\gamma \leq \gamma_{\text {des }} \\
\beta \geq \beta_{\text {des }}
\end{gathered}
$$

where $\theta_{\ell}$ are the corners of the uncertainty hypercube.

So far, the uncertainties considered have been those associated with the plant model. However, possible uncertainties in the actuators, due to degradation or failure, also need to be considered. The approach here is to consider actuator faults or failures one at a time. In other words, the control design considers only individual faults or failures in the actuators and the nominal controller must provide tolerance against these failures. The LMI framework can easily accommodate the one-at-a-time fault or failure approach. This is accomplished by adding an $H_{\infty}$ LMI (similar to Eq. (21)) for each actuator, i.e.,

\begin{tabular}{|l|l|}
\hline Length & $15 \mathrm{~m}$ \\
Mass & $101.25 \mathrm{Kg}$ \\
Inertia & $1898.44 \mathrm{Kg} \mathrm{m}{ }^{2}$ \\
1st Modal Frequency & $7.29 \mathrm{Rad} / \mathrm{s}$ \\
1st Modal Damp. Ratio & $0.1 \%$ \\
2nd Modal Frequency & $20.11 \mathrm{Rad} / \mathrm{s}$ \\
2nd Modal Damp. Ratio & $0.1 \%$ \\
\hline
\end{tabular}

Table 1 System Properties

$$
\begin{aligned}
& {\left[\begin{array}{c}
A\left(\theta_{\ell}, \delta_{i}\right) X+B\left(\delta_{i}\right) \hat{C}+(*)^{T} \\
\hat{A}+A^{T}\left(\theta_{\ell}, \delta_{i}\right)+C^{T} \hat{D}^{T} B^{T}\left(\delta_{i}\right) \\
H^{T}+F_{w}^{T} \hat{D}^{T} B^{T}\left(\delta_{i}\right) \\
L X+E_{z} \hat{C}
\end{array}\right.} \\
& \text { (.) } \\
& \begin{array}{c}
Y A\left(\theta_{\ell}, \delta_{i}\right)+\hat{B} C+(*)^{T} \quad \ldots \\
H^{T} Y+F_{w}^{T} \hat{B}^{T} \\
L+E_{z} \hat{D}
\end{array} \\
& \left.\begin{array}{cc}
(\cdot) & (\cdot) \\
(\cdot) & (\cdot) \\
-\alpha \gamma I_{w} & (\cdot) \\
C D_{z w}+E_{z} \hat{D} F_{w} & -\alpha \gamma I_{z}
\end{array}\right]<0
\end{aligned}
$$

where $\delta_{i}, i=1, \ldots, m_{i}$ denotes the corners of the uncertainty hypercube associated with actuator No. " $i$ " fault or failure, and $\alpha$ is a scalar which defines the acceptable level of performance degradation from the fully operational system with no hardware failures. A value of $\alpha=1$ dictates that the same level of performance is required from the system with one failed actuator as the fully operational system. Typically, $\alpha$ should be chosen greater than 1 .

\section{Numerical Results}

The proposed approach for fault-accommodating control is applied to the control synthesis of a twodimensional flexible system, as shown in Figure 2. The inertia and geometric properties of the system along with the frequencies and damping ratios of its first two flexible modes (used in the control synthesis) are provided in Table 1.

\section{Control System Hardware and Setup}

As shown in Figure 2, there are three control inputs available to provide fine pointing for the system. These inputs are three reaction wheels, one at the left end $(x=0 \mathrm{~m})$, one at the mid-length $(x=7.5 \mathrm{~m})$, and one at the two-thirds of the length $(x=10 \mathrm{~m})$. It is assumed that attitude measurements are available from a set of three collocated attitude sensors. Here, sensors and actuators dynamics are not considered. There is an exogenous disturbance source (force disturbance) at one third of the length $(x=5 m)$. The performance of the system is measured in terms of the attitude pointing error at the right end $(x=15 m)$. As mentioned 
earlier, the control design is based on $H_{\infty}$ synthesis. Figure 1 shows the closed-loop block diagram. A 6thorder model of the system, which included the first two flexible modes, were used in the control design.

The performance requirements were defined in terms of the weighted output sensitivity transfer function from the disturbance to the performance output. The weighting function was chosen as $W_{p}=\frac{50(s+6 \pi)}{(s+0.06 \pi)}$ to provide good disturbance rejection at low to mid frequencies. Uncertainty in the system model in the form of unstructured input multiplicative was used. The weighting function, used to scale or distribute the uncertainty, was chosen as $W_{u}=\frac{0.1(s+0.05)}{(s+500)}$ to emphasize model uncertainty in the high frequency range. As for the structured plant uncertainty, a $5 \%$ uncertainty in the modal frequency of the first two modes were assumed.

To accommodate the potential uncertainties in the actuators due to degradation or failure, additional dynamics (in the form of a fictitious actuator dynamics) were augmented with the plant dynamics. The aug mented dynamics were as follows for each control input channel

$$
A_{a}=-100 ; B_{a}=100 ; C_{a}=1 ; D_{a}=0
$$

Uncertainties $\delta_{1}$ and $\delta_{2}$ were considered for the matrices $B_{a}$ and $A_{a}$, respectively, to account for the potential actuator fault/failure. $\delta_{1}$ was chosen to vary from 0 to 1 to allow for a variation from nominal performance to total failure, and $\delta_{2}$ was chosen to vary from 1 to 10 . The nominal augmentation and its associated uncertainties were chosen such that they provide a range of perturbations in the system due to actuator degradation/failure. These perturbations run the garnut from full actuator operation (with no impact on plant dynamics) to the total failure of any one actuator, with potential gain loss and/or phase delays in between. For these failures the design required the system to remain stable, but allowed an increase of up to a factor of five in $H_{\infty}$ norm constraint, i.e., $\alpha=5$.

\section{Control Synthesis}

The LMI framework, described in the previous section, was used to synthesize a fault-accommodating controller. The controller was to provide a desired $H_{\infty}$ norm performance for the weighted output sensitivity transfer function from disturbances to the performance output, while tolerating uncertainties in the modal frequencies of the first two flexible modes, and allowing for degradation or total failure of any one of the three reaction wheels. As indicated earlier, these requirements reduce to the solution of the system of LMIs given in Eqs. (21)-(25). These LMIs were solved using the LMI Control Toolbox. ${ }^{8}$ The design parameters $\beta_{\text {des }}$ and $\gamma_{\text {des }}$ were chosen to be 1.25 and 1.0 , respectively. The LMI feasibility problem was solved using the "feasp" routine of the LMI Toolbox. ${ }^{8}$
The singular value plot for the resulting controlled system is given in Figure 3. Figure 3a shows the singular values of the open and closed-loop system over the full path, including both performance and robustness conditions. Figure $3 \mathrm{~b}$ show performance alone, comparing the disturbance rejection of the exogenous disturbance for the open-loop and closed-loop system. It is observed that the $H_{\infty}$-based controller provides good disturbance rejection at low-mid frequencies, including the first flexible mode. However, this is not the case above the first flexible frequencies because of the uncertainties associated within the modal frequency and the ramp-up of actuator input uncertainty.

To validate the robustness of the system against real parametric uncertainties the controller was applied to a set of plant models which had randomly chosen frequency errors within the $5 \%$ bound specified in the design. A histogram of the resulting $H_{\infty}$ norm for these systems is shown in Figure 4 and shows very little variation in performance due to these errors.

In Figure 5 singular values plots are shown for the closed-loop systern subject to hard failures in each of the actuators. In can be seen that actuator-3 is the most critical to maintaining performance, while the loss actuator-1 has little impact. All cases however, retain a level of performance as specified by the design constraints. This observation is also borne out in the $H_{\infty}$ norms shown in Table 2. Finally, the fault tolerance of the controller was also demonstrated by simulation. A linear simulation of the system response, subject to band-limited white noise disturbance, was performed for each failure case. Figure 6 shows the system under operational conditions, and with the failure of each actuator in turn in for 1000 second durations.

\section{Concluding Remarks}

Control of flexible systems subject to degradation or failure of sensors/actuators was considered. A Linear Matrix Inequality framework was developed to synthesize $H_{\infty}$-based controllers, which provide good disturbance rejection while capable of tolerating real parameter uncertainties in the system model, as well as potential degradation or failure of the control system hardware. In this approach, the controller can accommodate faults or failures in one sensor or actuator at any given time. This is a reasonable approach for most aerospace applications, which typically have limited number of highly reliable components. A numerical example involving the control synthesis for a two-dimensional flexible system was worked out to demonstrate the feasibility of the proposed approach. The results demonstrated the effectiveness of the fault accommodating control strategy as well as the feasibility of LMI-based approach for multi-objective synthesis problems. 


\section{References}

${ }^{1}$ Balas, G. J. and Doyle, J. C., "Robustness and Performance Tradeoffs in Control Design for Flexible Structures," IEEE Control Systems, Vol. 2, No. 4, 1994, pp. 352-361.

${ }^{2}$ Joshi, S. M. and Maghami, P. G., "Robust Dissipative Compensators for Flexible Spacecraft Control," IEEE Transactions on Aerospace and Electronic Systems, Vol. 28, No. 3, 1991, pp. $768-774$.

${ }^{3} \mathrm{Lim}$, K. B., Joshi, S. M., and Maghami, P. G., "Comparison of Controller Designs for an Experimental Flexible Structure," IEEE Control Systems, 1992, pp. 108-118.

${ }^{4}$ Maghami, P. G., Sparks, Jr., D. W., and Lim, K. B., "Fault Accommodation in Control of Flexible Systems," AIAA, Journal of Guidance, Control, Dynamics, Vol. 21, No. 3, 1998, pp. 500-507.

${ }^{5} \mathrm{El}$ Ghaoui, L. and Niculescu, S.-L., Adavences in Linear Matrix Inequality Methods in Control, Society for Industrial and Applied Mathematics, 2000.

${ }^{6}$ Scherer, C., Gahinet, P., and Chilali, M., "Multiobjective Output-Feedback Control via LMI Optimization," IEEE Trans actions on Automatic Control, Vol. 42, No. 7, 1997, pp. 896-911.

${ }^{7}$ Apkarian, P. and Adams, R. J., "Advanced Gain-Scheduling Techniques for Uncertain Systems," IEEE Transactions on Control Systems Technology, Vol. 6, No. 1, 1998, pp. 21-32.

${ }^{8}$ Gahinet, P., Nemirovski, A., Luab, A., and Chilali, M., LMI Control Toolbox, The MathWorks, Inc., 1995.

\begin{tabular}{|l|c|c|}
\hline Case & $\left\|\mathbf{G}_{\mathbf{z w}}\right\|_{\infty}$ & $\left\|\mathbf{G}_{\mathbf{p d}}\right\|_{\infty}$ \\
\hline Fully Operational & 0.482 & 0.069 \\
Hard Failure, Actuator- 1 & 0.479 & $\mathbf{0 . 0 6 4}$ \\
Hard Failure, Actuator-2 & 0.551 & $\mathbf{0 . 1 5 6}$ \\
Hard Failure, Actuator-3 & $\mathbf{1 . 9 9 3}$ & $\mathbf{0 . 9 8 9}$ \\
\hline
\end{tabular}

Table $2 H_{\infty}$ norms for coupled Robustness and Performance path, $G_{z w}$, and for performance path alone, $G_{p d}$, under single-fault hard failures.

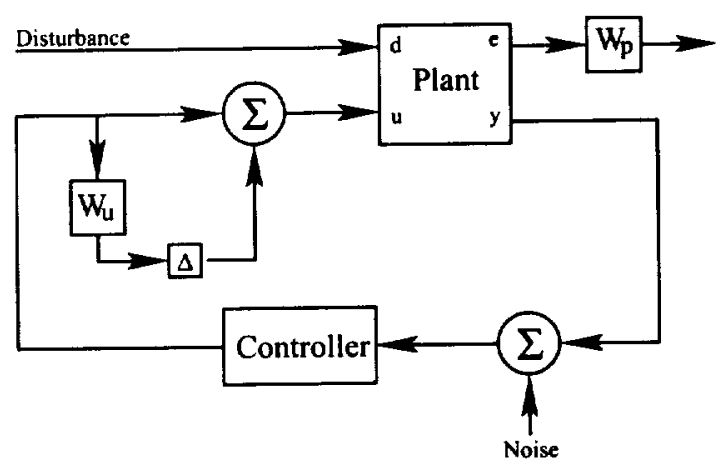

Fig. 1 Block diagram of the $H_{\infty}$ problem

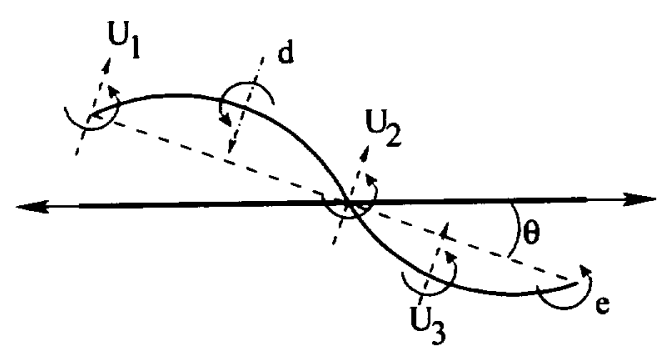

Fig. 2 Schematic of the two-dimensional flexible system 


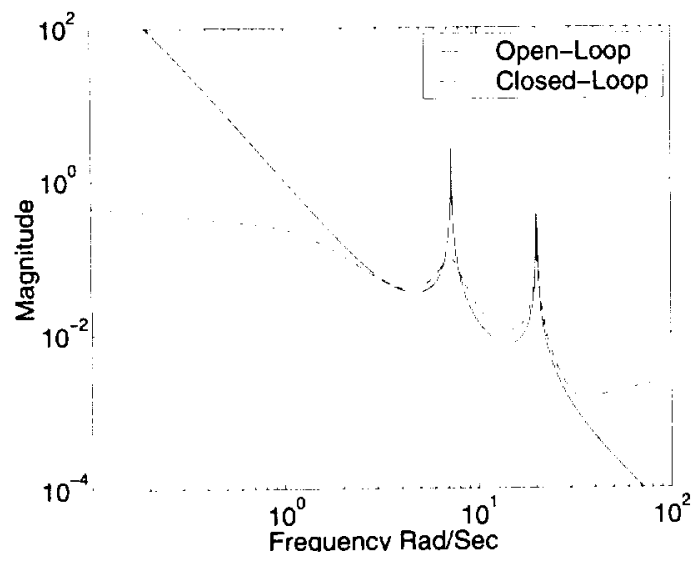

(a) Fully coupled performance/robustness path

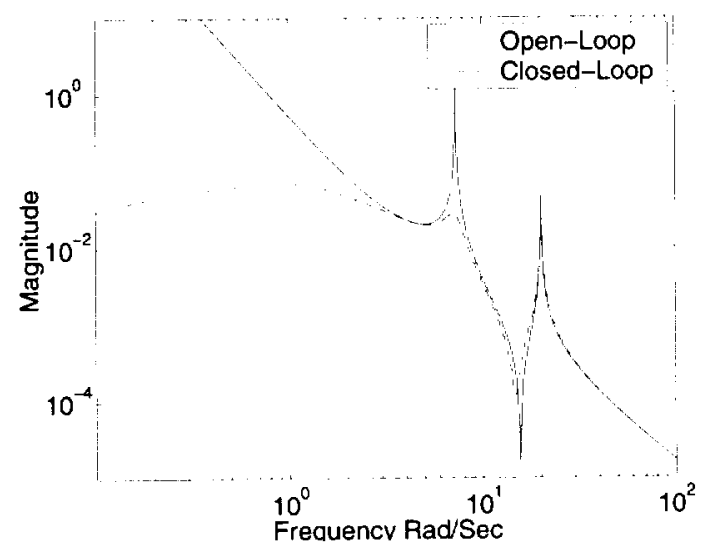

(b) Performance path only

Fig. 3 Maximum singular value plot for open and nominal closed loop system.

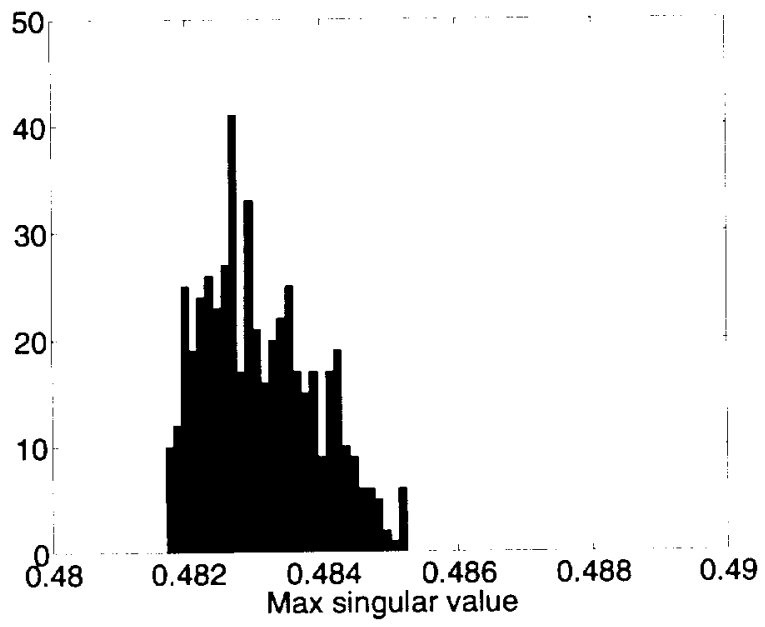

Fig. 4 Variation of $H_{\infty}$ norm with $5 \%$ errors in natural frequency, 500 cases

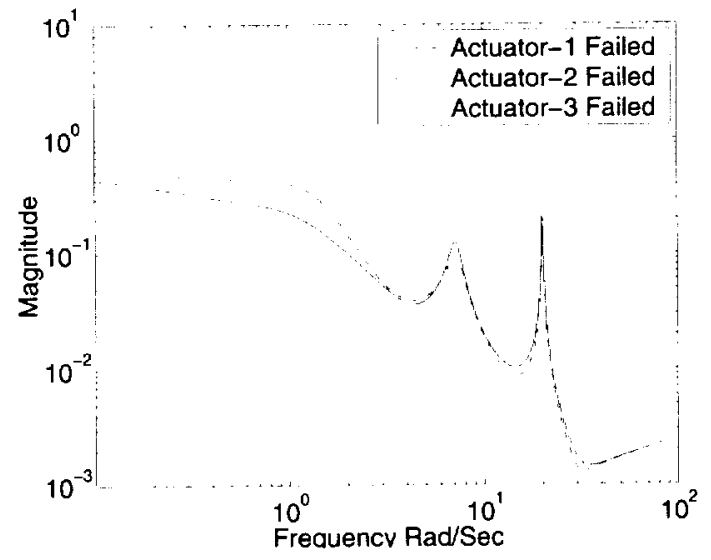

(a) Fully coupled performance/robustness path

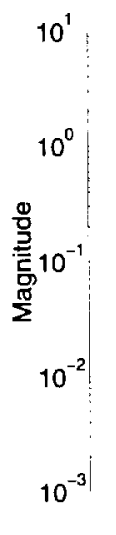

Actuator-1 Failed Actuator-2 Failed Actuator-3 Failed

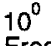

Frequency Rad/Sec

(b) Performance path only

Fig. 5 Maximum singular value plot for closedloop system under different actuator failures

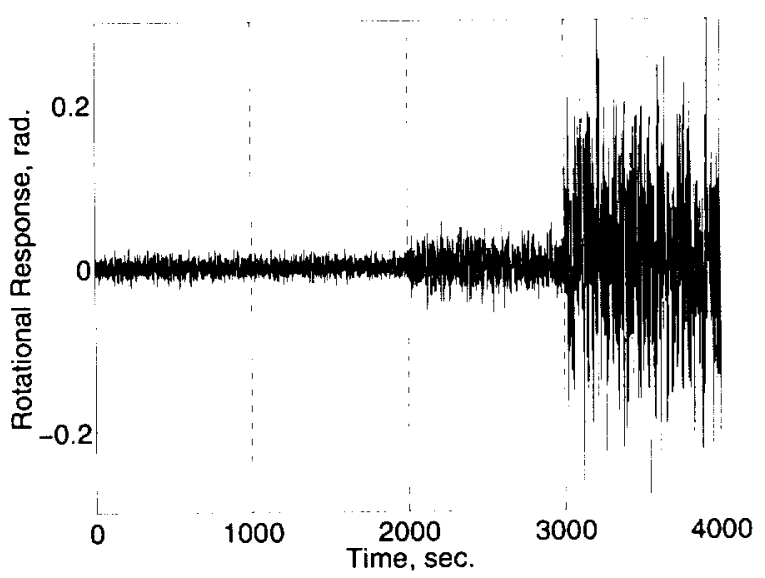

Fig. 6 Time response of pointing under disturbance for four conditions: Operational, Act-1 Failure, Act-2 Failure, and Act-3 Failure 